\title{
Análisis de natalidad y mortalidad en Tungurahua.
}

\section{Introducción}

La natalidad y mortalidad, son indicadores demográficos que permiten conocer la dinámica y la evolución de los fenómenos estadísticos de nacimientos y defunciones que acontecen en el país y provincia. Su objetivo, es obtener un conocimiento adecuado sobre las principales características y causas que influyen en el desarrollo demográfico de la población.

Los datos proporcionados, brindan un apropiado entendimiento de cómo el fenómeno demográfico ha ido variando con el paso del tiempo la población del país, aspectos sumamente importantes en el crecimiento estructural a nivel nacional y de Tungurahua.

En la figura 1, se observa el número de nacidos vivos durante los últimos 10 años, aquí se incluye los nacidos vivos oportunos (nacimientos ocurridos e inscritos en el año de estudio) y tardíos (nacimientos ocurridos en el año de estudio pero inscritos en el año subsiguiente), su evolución y detalle por género, donde se destaca un decrecimiento de nacimientos en los años 2009, 2011 y 2012, siendo este último año, el que registra mayor disminución con un $7,09 \%$, en relación al 2011; en el caso del 2014 se toman tan solo los datos de los nacidos vivos oportunos por lo que no se puede realizar un análisis real para determinar si los nacimientos se han incrementado o disminuido con respecto al 2013.

Figura 1: Número de nacidos vivos por género a nivel nacional Periodo 2005 - 2014

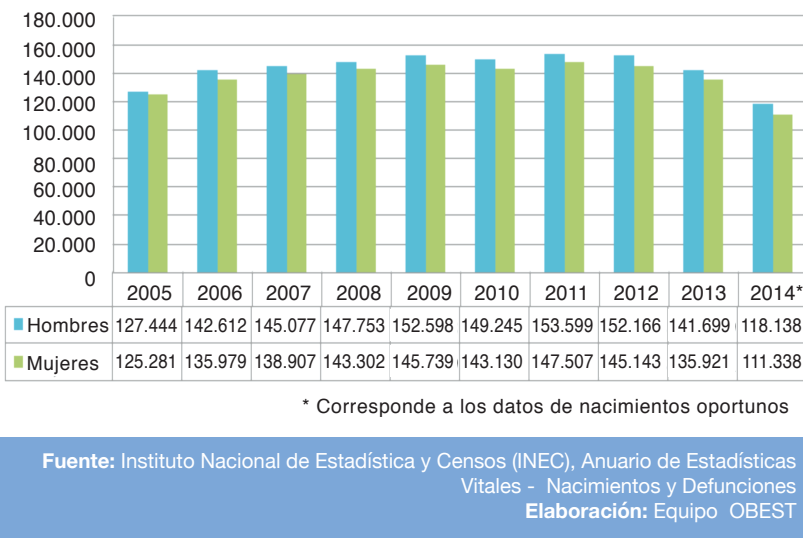

En la figura 2, se refleja los lugares donde las madres acuden a dar a luz, se evidencia que la utilización de establecimientos públicos y privados durante los 3 últimos años ha ido disminuyendo; el Instituto Ecuatoriano de Seguridad Social - IESS ha incrementado el número de nacimientos en una mínima proporción.

Durante los años 2012 - 2014, el 50,5\% del total nacional, han utilizado el servicio de las instituciones públicas, seguido de instituciones privadas con el $28,5 \%$; el $7,9 \%$, dan a luz en casas, el $6 \%$ se atiende en el IESS.
Figura 2: Nacidos vivos por lugar de ocurrencia a nivel nacional Periodo $2012-2014$

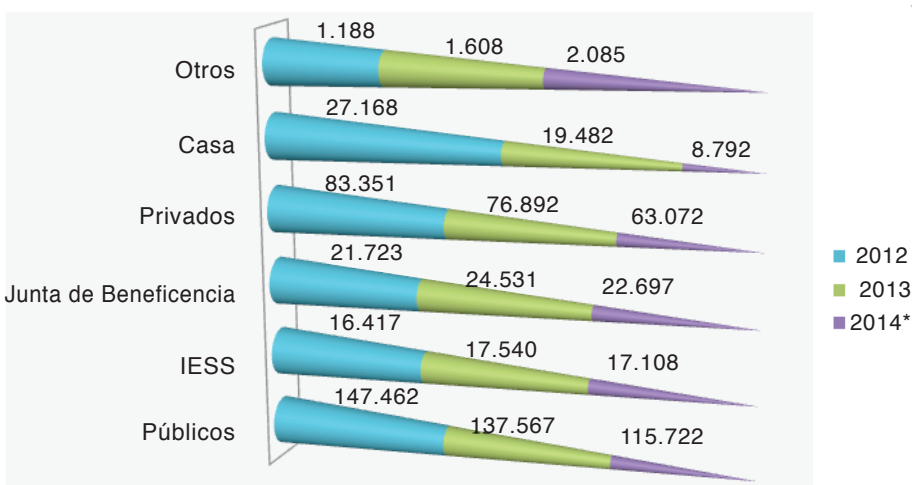

* El dato será ajustado con los nacimientos ocurridos en el año 2014 e inscritos en el año 2015

Fuente: Instituto Nacional de Estadística y Censos (INEC), Anuario de Estadísticas Vitales Nacimientos y Defunciones Elaboración: Equipo OBEST

La figura 3, refleja datos respecto a las mujeres de entre 20 y 24 años, donde se identifica el mayor rango de natalidad, asistidas con personal médico y no médico, adicional, se puede destacar que entre las edades de 15 y 19 años el número de nacimientos es de 39.772 con asistencia médica y 1.679 sin asistencia médica, representa el $18 \%$ del total nacional.

Figura 3: Nacimientos a nivel nacional, según rango de edad de la madre y según asistencia de personal médico en el parto. Año 2014

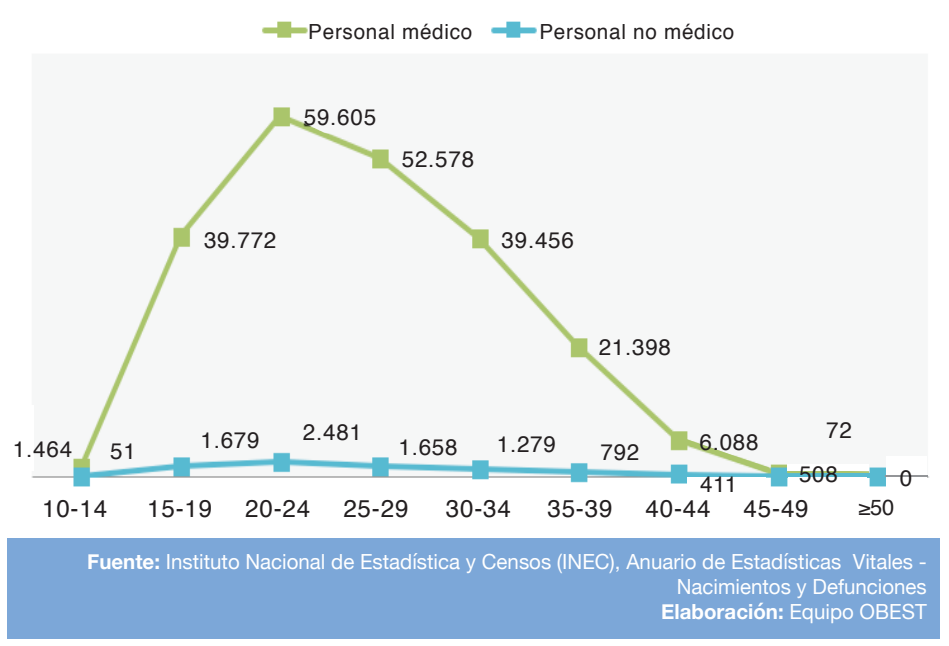

La figura 4, determina que durante el periodo de 1990 -2014 las muertes infantiles (menores de un año) y de niñez (menores de cinco años) disminuye cada año, esto debido a varios factores como son: el incremento de la cobertura y, un correcto sistema articulado para la vacunación ya que a partir de 1997, el Ministerio de Salud Pública, a través del Programa Ampliado de Inmunizaciones cuenta con la Ley de vacunas, el mismo que garantiza el abastecimiento oportuno y permanente de vacunas. 
Figura 4: Número de muertes infantiles y niñez a nivel nacional Periodo 1990 - 2014

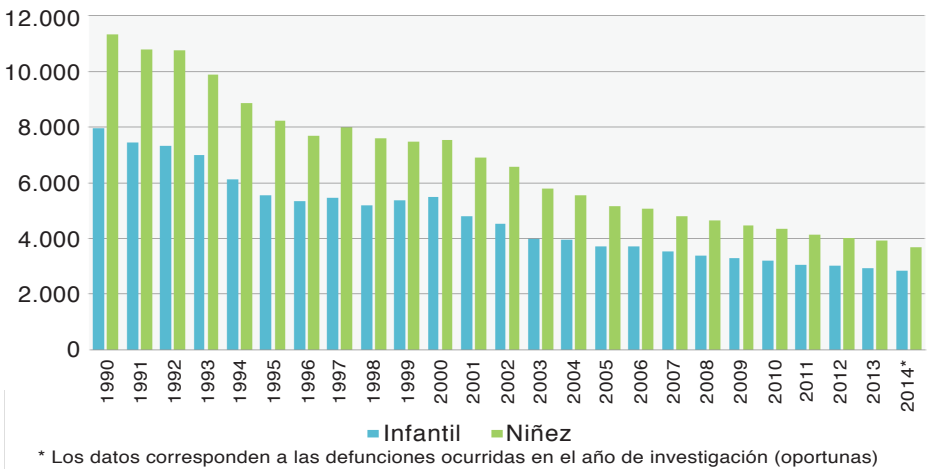

Fuente: Instituto Nacional de Estadística y Censos (INEC), Anuario de Estadísticas
Vitales - Nacimientos y Defunciones
Elaboración: Equipo OBEST

En la figura 5, se representa la tasa de mortalidad general y la tasa de mortalidad infantil (menores de un año) durante el periodo 20042014; durante estos años, la tasa de mortalidad más baja corresponde al 2014 con 8,35 y 3,93 respectivamente.

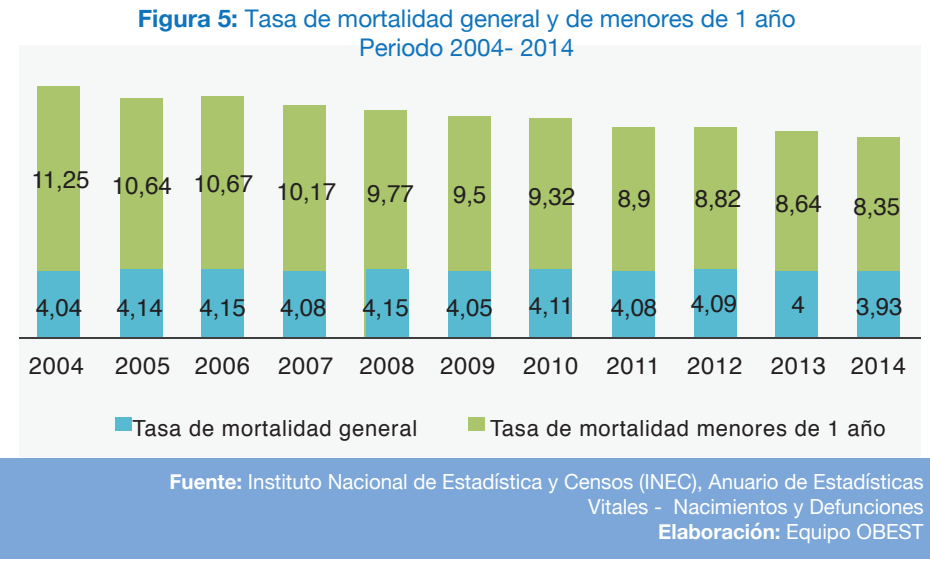

La figura 6, aborda el Indicador de Razón de Muerte Materna RMM (número anual de mujeres fallecidas por causas relacionadas con el embarazo y el parto por cada 100.000 nacidos vivos), por lo tanto la figura ya mencionada, establece que la provincia que mayor RMM tiene es Zamora Chinchipe con 142,20 es decir, que las mujeres que se encuentran en esta provincia tienen mayor riesgo obstétrico de morir durante la gestación.

En Tungurahua durante el 2014 existió 4 muertes maternas; y, el RMM es de 37,69 lo que indica que aproximadamente 38 mujeres tungurahuenses mueren por cada 100.000 nacimientos. A nivel nacional, para el año 2014 se registraron 166 muertes maternas, dando como resultado un RMM de 49,16, lo que significa que por cada 100.000 nacimientos, fallecen aproximadamente 49 mujeres en edad fértil, la meta que el gobierno se plantea es reducir la muerte materna, para de esta manera cumplir con los objetivos del milenio, por lo que se está realizando fuertes inversiones en hospitales y centros de salud que brinden un buen servicio gineco-obstétrico a nivel nacional.
Figura 6: Indicador razón de muerte materna RMM, según provincias de residencia habitual. Año 2014

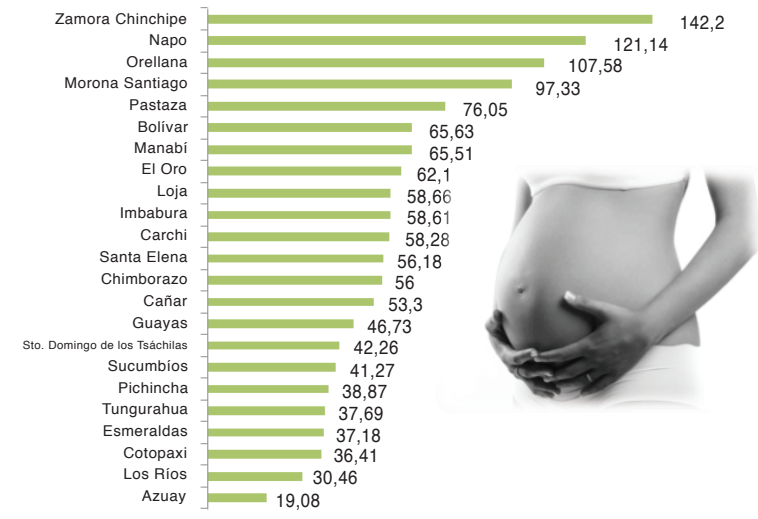

Fuente: Instituto Nacional de Estadística y Censos (INEC), Anuario de Estadísticas Vitales - Nacimientos y Defunciones Elaboración: Equipo OBEST

En la figura 7, se identifica las provincias con mayor número nacimientos inscritos durante el 2014, siendo Pichincha la provincia con más nacimientos durante este año con 41.991; a continuación esta Azuay 11.528; Tungurahua 7.901 lo que representa un $8 \%$ de los nacimientos a nivel nacional; Santo Domingo de los Tsáchilas 6.760, Chimborazo 7.243; Loja 6.575 y finalmente 21.023 nacimientos se distribuyen entre el resto de provincias de la región sierra.

Figura 7: Nacidos vivos por provincias de la región sierra, inscritos en el año 2014

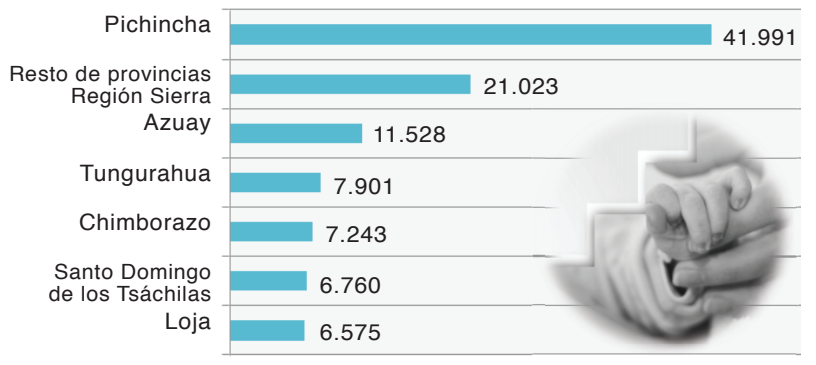

Fuente: Instituto Nacional de Estadística y Censos (INEC), Anuario de Estadísticas Vitales - Nacimientos y Defunciones Elaboración: Equipo OBES

Figura 8: Nacimientos en Tungurahua según zona urbana y rural. Año 2014
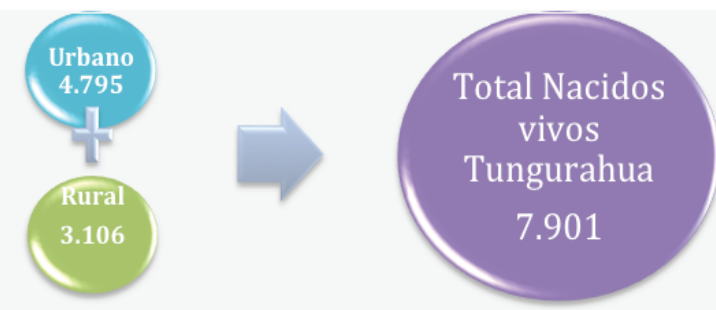

Fuente: Instituto Nacional de Estadística y Censos (INEC), Anuario de Estadísticas Vitales - Nacimientos y Defunciones Elaboración: Equipo OBEST 
La figura 9, describe los nacimientos y defunciones generales en Tungurahua por cantones, donde destaca Ambato con 6.810 nacimientos lo que representa el $86 \%$ a nivel provincial; Pelileo el 1\%; y, el resto de cantones se divide los 549 nacimientos el 13\%; en defunciones podemos destacar que en Quero y Baños existe mayor número de defunciones que nacimientos durante el 2014, además, cabe indicar que en Ambato por cada 6.810 nacimientos existen 4 muertes, en Pelileo por cada 598 nacido vivos mueren 2 personas, algo totalmente diferente sucede en Quero y Baños donde por cada 105 o 104 muertes ocurre un nacimiento.

Figura 9: Nacimientos y defunciones generales por cantones en Tungurahua Año 2014

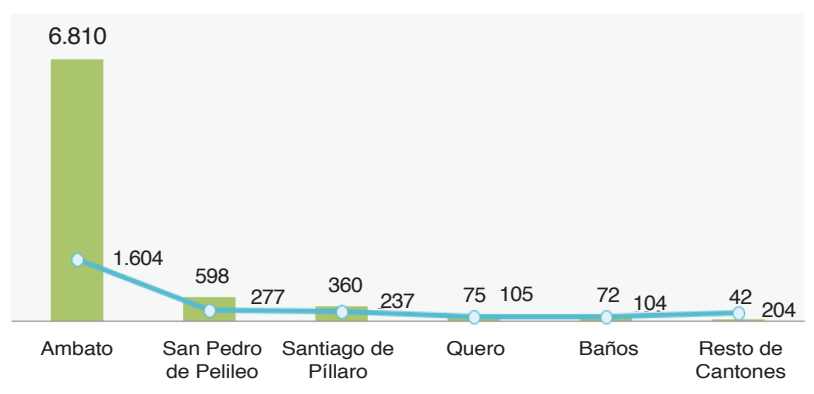

Nacimientos $\rightarrow-$ Defunciones

Fuente: Instituto Nacional de Estadística y Censos (INEC), Anuario de Estadísticas Vitales - Nacimientos y Defunciones Elaboración: Equipo OBEST

La figura 10, indica que en Tungurahua el $56 \%$ de los nacimientos son de madres casadas, el $32 \%$ solteras; el $9 \%$ unidas y el $3 \%$ se distribuye entre el resto de estados civiles (separada, divorciada, viuda).

Figura 10: Nacidos vivos, por estado civil de la madre en Tungurahua Año 2014

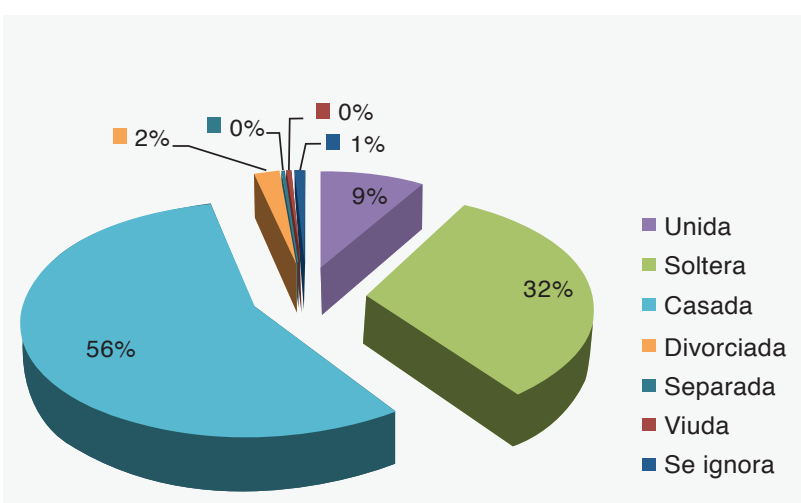

Fuente: Instituto Nacional de Estadística y Censos (INEC), Anuario de Estadísticas Vitales - Nacimientos y Defunciones Elaboración: Equipo OBEST
La figura 11, representa el nivel de instrucción de las madres en Tungurahua, el mayor número de nacimientos son de progenitoras que cursan la primaria, lo que representa el $27,5 \%$; el $25,6 \%$ son madres en secundaria con 2.025 nacimientos; y mujeres que terminan sus estudios superiores el $20,5 \%$; las madres que no saben leer ni escribir el 1,7\% con 132 nacimientos.

Figura 11: Nacidos vivos, por nivel de estudios de la madre en Tungurahua Año 2014

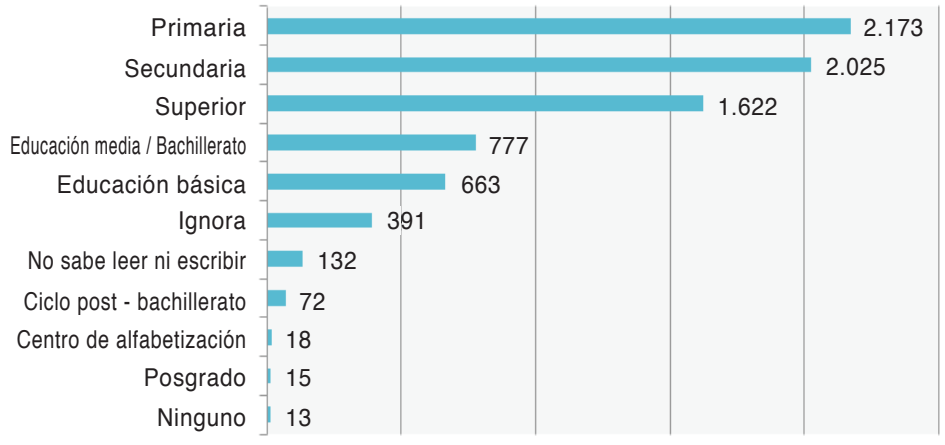

Fuente: Instituto Nacional de Estadística y Censos (INEC), Anuario de Estadísticas Vitales - Nacimientos y Defunciones Elaboración: Equipo OBEST

Con los datos analizados se da a conocer la realidad del país con respecto a los nacimientos y defunciones, además datos importantes como rangos de edades de mujeres que tienen hijos, su estado civil y nivel de instrucción.

Referencias:

- Instituto Nacional de Estadística y Censos (INEC), Anuario de Estadísticas Vitales Nacimientos y Defunciones.

Elaboración:

Econ. Tatiana Vayas 